\title{
GRBSpec and GRBPhot: social networks to share gamma ray burst data
}

Blažek, Martin, de Ugarte Postigo, A., Kann, D., Thöne, C. C., Aguíi Fernandez, J., et al.

Martin Blažek, A. de Ugarte Postigo, D. A. Kann, C. C. Thöne, J. F. Aguí Fernández, L. Izzo, "GRBSpec and GRBPhot: social networks to share gamma ray burst data," Proc. SPIE 11452, Software and Cyberinfrastructure for Astronomy VI, 1145218 (13 December 2020); doi: 10.1117/12.2562420

SPIE. Event: SPIE Astronomical Telescopes + Instrumentation, 2020, Online Only 


\title{
GRBSpec and GRBPhot: Social networks to share Gamma Ray Burst data
}

\author{
Martin Blazek $^{\mathrm{a}}$, A. de Ugarte Postigo ${ }^{\mathrm{a}, \mathrm{b}}$, D. A. Kannª , C. C. Thöne ${ }^{\mathrm{a}}$, J. F. Agüí Fernández ${ }^{\mathrm{a}}$, \\ and L. Izzo ${ }^{b}$ \\ aAA-CSIC, Glorieta de la Astronomía, s/n, 18008 Granada, Spain \\ ${ }^{\mathrm{b}}$ DARK, Niels Bohr Institute, University of Copenhagen, Lyngbyvej 2, DK-2100 Copenhagen \\ $\varnothing$, Denmark
}

\begin{abstract}
GRBSpec and GRBPhot are two databases designed for the storage and analysis of gamma-ray burst (GRB) data. GRBSpec is devoted to spectroscopic observations, GRBPhot to photometric data. Both databases have a detailed search engine and offer online graphical tools for plotting and data analysis. They aim to publicly share these specialised data among the astronomical community and provide quick online measurements and plots. The databases can be accessed through http://grbpsec.iaa.es and http://grbphot.iaa.es, respectively. As of November 2020, the database already contained 2013 files belonging to 810 spectra of 268 different GRBs.
\end{abstract}

Keywords: spectroscopy, photometry, database, gamma-ray burst

\section{INTRODUCTION}

Gamma-ray bursts (GRBs) are some of the most energetic events that we can observe in the Universe. Due to their luminosity, they have been observed at almost any distance, from the present, local Universe, to the very distant, at the epoch of reionization. The study and theoretical research of GRBs has been an essential part of high-energy astrophysics during the last decades. GRBs are generally divided into two classes: long GRB with soft spectra are produced during the collapse and explosion of massive stars, usually accompanied by the presence of a broad-lined Ic supernova, ${ }^{1}{ }^{2}$ Short GRBs occur after the merger of two compact objects (neutron star and black hole) and are connected with gravitational-wave emission. ${ }^{3}$ As the number of observed GRBs grow, detailed observations have shown the possible existence of further, unique groups, such as long GRBs without associated supernova, ${ }^{4}$ ultra-long GRBs associated with extremely bright supernovae, ${ }^{5}$ or black-body dominated GRBs. ${ }^{6}$ Due to their very rapid evolution, it was necessary to construct fast-response instruments both in space and on the ground. Much of the most valuable observations need to be acquired during the first minutes/hours after the event. With the era of fast triggering X-ray $/ \gamma$-ray space satellites and world telescope network cooperation, the information about this phenomena now reaches the point where not only a large amount of photometric data is available, but still more and more spectral data from the large telescopes have been collected. Here we present the online databases and analysis tools GRBSpec* and GRBPhot ${ }^{\dagger}$, which have been conceived to simplify the sharing of the spectral and photometric data among the community and to guarantee their availability. GRBSpec is a collaborative database containing processed spectra from various observatories. It provides both visualization and analysis tools for multiple data formats and origins and simplifies sharing GRB data with the community. Historically, it evolved from a small project ${ }^{7}$ in 2013 and now nearly 100 high-energy users from all around the world are registered and taking advantage. The development of GRBPhot started in 2019 (with data collection beginning in the early 2000s) and used the core of the GRBSpec GRB information. It is based on the optical afterglow sample that has been used in multiple papers ${ }^{8-11}$ and is continually being expanded (Kann et al. 2021a,b, in prep.). It represents the largest optical photometry database of GRBs in the world, containing over 20,000 data points at this time.

Both databases offer the following features and tools, among others:

\footnotetext{
Further author information: (Send correspondence to M.B.)

M.B.: E-mail: alf@iaa.es

*http://grbspec.iaa.es

${ }^{\dagger}$ http://grbphot.iaa.es
} 
- Database query by GRB or observation properties $\left(T_{0}, T_{90}\right.$, satellite name, spectral resolution, Signal-tonoise-ratio, coordinates, etc.)

- Data upload/download in various formats, including ASCII, FITS ${ }^{12}$ or those used by the Stargate collaboration for X-shooter ${ }^{13}$

- Storing of data tables of equivalent widths (EWs), fluxes and column densities

- Graphical applets for both 1D and 2D visualization of spectra (GRBSpec) or light curves (GRBPhot)

- Measuring tool for EWs

- Multiplotting tool for the comparison of several spectra

- Tool for the animation of several spectra together (e.g., time evolution display)

- 'Sandbox' mode for private data plotting, measurement, or redshift determination

- Possibility of creating scientific groups/collaborations with whom to share private data

- Each uploader is the owner of their data and can decide who can view/modify/erase the data and the results

- Automatic determination of line strength diagrams.

\section{DATABASE STRUCTURE AND WEB DESIGN}

The databases are based on MySQL ${ }^{\ddagger}$ technology for storing information and querying. The relations between tables and SQL representation were designed with a focus on the spectral data. The structure of the database is prepared for the further development of features such as storing and analyzing of spectral features. The system stores data in FITS format ${ }^{12}$ which are used for visualization and analysis, while other formats are accepted for upload or download. The expected format of the spectrum can be one or two-dimensional, flux calibrated, normalized, or in counts and can include errors. The connection between server and client is done using Apache and PHP hypertext preprocessor scripts ${ }^{\S}$. The access to the data is secured using five levels of authorization from guest to administrator's rights using a hash. Data themselves cannot be accessed directly from outside the server without logging in. Both databases are free for the community and the users are just required to register to ensure the security and integrity of the database. The web access $\mathbb{I}$ offers combined search queries, user management, uploading and downloading of data, links to external sources (telescopes, instruments, and ADS articles), as well as dozens of additional data related to each spectrum to allow searching, spectral visualization, and spectral analysis.

\subsection{Data format}

As of November 2020, the database contained 3.3 gigabytes of processed data (excluding raw files). The following data formats are accepted:

- Astronomical binary table in FITS format ${ }^{12}$ for both uploading and downloading,

- ASCII text files in 3-column format separated by space or tabulator containing wavelength (in laboratory frame), the spectrum itself and error spectrum

- ASCII text files in 8- or 9-column format separated by space or tabulator which follows the format used by the Stargate collaboration for X-shooter at the VLT telescope ${ }^{13}$ (including wavelengths in both air or vacuum, flux, error, and a slit-loss parameter).

\footnotetext{
${ }^{\ddagger}$ http://www.mysql.com

$\S$ http://www.php.net

$\mathbb{I}_{\text {http: / /grbspec.iaa.es }}$
} 
The database can store the following data:

- One-dimensional or two-dimensional count spectrum or count error spectrum,

- One-dimensional or two-dimensional flux-calibrated spectrum or flux-calibrated error spectrum,

- One-dimensional normalized spectrum or normalized error spectrum,

- Simple image of spectrum for fast preview

- Zipped auxiliary files (acquisition, calibration, ...).

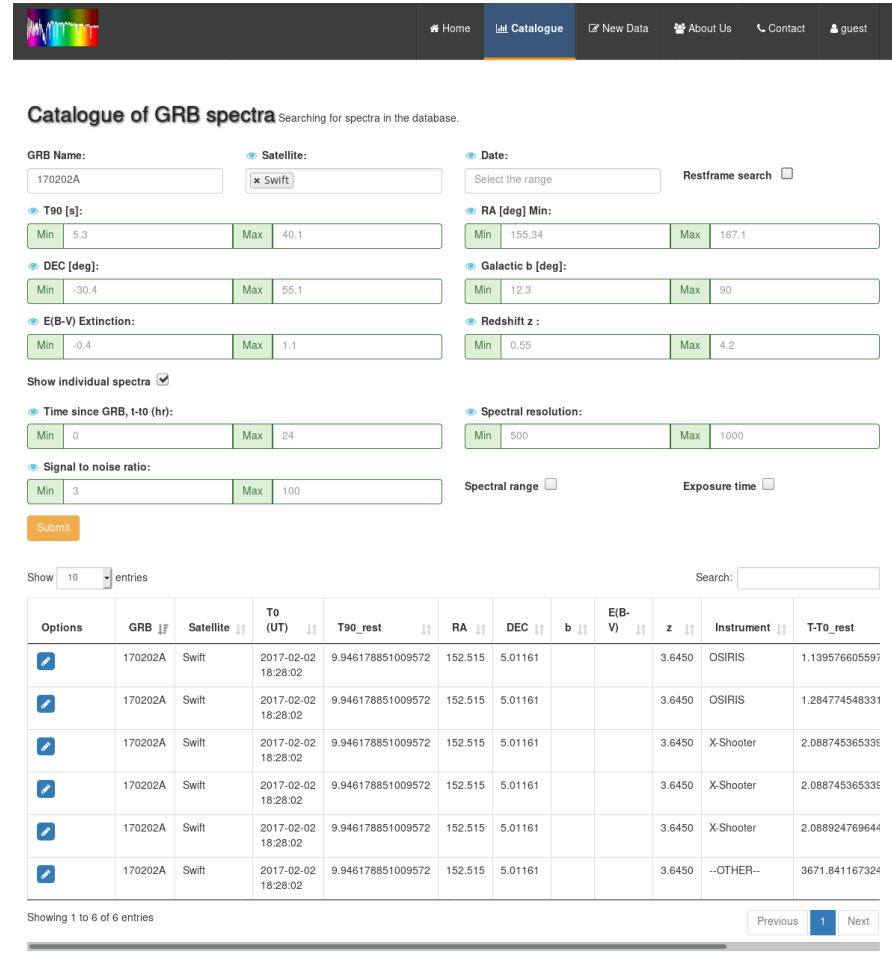

Figure 1. Search engine of GRBSpec.

\subsection{Online access}

After logging in, the main menu is divided into several sections. The content of the database can be queried in the 'Catalogue' menu (an example of the search engine is shown in Fig. 1). Inside the 'New Data' section, the user can upload their data to the database or manually create a new GRB (if it has not been previously found from the SWIFT/INTEGRAL/Fermi automated update), or the user can enter the Sandbox mode. The Sandbox allows the user to use the features and tools of GRBSpec without influencing the real catalog, such as plotting, redshift determination, or measurement of spectral features in private data. Data, files and results uploaded to this sandbox mode are invisible to any other user and can be easily modified, uploaded, or erased without fear of losing or erasing any data in the real catalog.

The real spectra of GRBs themselves can be accessed through the main section 'Catalogue', which leads to the search engine (see Fig. 1). Clicking on a row of the result table leads to an overview of the selected GRB with information about coordinates, $T_{0}, T_{90}$, measured redshifts $z$ (for the GRB and foreground absorbers), fluence, and to the list of individual spectra or light curves associated with this GRB. Spectra can be independent or form observational datasets (for instance, if obtained simultaneously by the same instrument with multiple 
arms). Choosing the row of a specific spectrum leads to the main page related to it. An example spectrum of GRB 170202A obtained by the OSIRIS instrument at the Gran Telescopio Canarias (GTC) is shown in Fig. 2 and Fig. 3.

\subsection{Graphical Tools}

GRBSpec and GRBPhot were designed with two basic principles in mind - to share the spectra and light curves among the community and to facilitate the fast online analysis of those data. For this purpose, the database does not contain only the data themselves, but also many other important information on the individual GRBs and the results of the data analysis. The graphical tool is a powerful aid to the determination of the GRB redshift as it allows to plot the most common lines observed in GRB spectra, regulating their amount according to their typical strength. It allows to recalculate the appropriate wavelengths in vacuum or to display the spectra with different binning or Gaussian smoothing. The user can plot the data together with sky lines or telluric features, to avoid confusion of real and atmospheric features and to check the precision of the wavelength calibration.

The graphical tools are based on the Plot.ly platform". They serve for both the online data analysis and the data preview. They allow plotting of the 1D spectra (flux-calibrated, count, or normalized) together with the 2D spectra (if uploaded) and the error spectra, as well as interactive light curve plotting and filtering. Data preprocessing includes the recalculation of vacuum/air wavelengths, optional binning or Gaussian smoothing. Data can be compared to the list of telluric features (GEMINI instrument**) and to the list of sky lines, which were taken from the archives of GEMINI telescope ${ }^{\dagger \dagger}$ and UVES ${ }^{\ddagger \ddagger}$ instrument. The graphical tool allows to plot a list of laboratory absorption and emission lines. ${ }^{14}$ The user can adjust the number of the strongest lines to be plotted and to plot several different colored sets for different values of redshift.

The online analysis includes the measurement of the positions, equivalent widths and errors of spectral features. It requires the user to define continuum regions around the spectral feature. This continuum is defined by the user by choosing four points, two of them defining the first spectral range (preferably at one side of the spectral feature that we want to measure) and another two defining the second spectral range (preferably at the other side of the spectral feature). The continuum with the error fit (including the variance from the error spectrum when available) is calculated from those two regions. Once this is done, the user is able to obtain the measurement of the wavelength equivalent width and error of a spectral feature by just clicking on it. The results can be then directly stored in the database, if desired.

A further graphical tool allows the plotting of multiple spectra in a single figure. This can be accessed through the catalog search after selecting 'Show individual spectra'. This multiplot feature can be used to simultaneously display the different spectra or through an animated display, automatically showing one spectrum at at time.

\section{FUTURE WORK}

Both databases are continuously being enhanced both by new features and new data. Much of the current development is devoted to the tools of GRBPhot, and the common integration of the two databases. These features will include online SED diagrams and the combined plot of photometric and spectroscopic data.

\section{ACKNOWLEDGMENTS}

GRBSpec and GRBPhot have been made possible through the generous contribution of a 2016 Leonardo Grant for Researchers and Cultural Creators, BBVA Foundation. AdUP and CT acknowledge support from Ramón y Cajal fellowships (RyC-2012-09975, RyC-2012-09984). MB acknowledges funding associated to a personal técnico de apoyo fellowship (PTA2016-13192-I). DAK acknowledges support from Spanish National Research Project RTI2018-098104-J-I00 (GRBPhot). LI was supported by grants from VILLUM FONDEN (project number 16599 and 25501). JFAF acknowledge financial support from Ministerio de Ciencia, Innovación y Universidades fellowship (PRE2018-086507).

\footnotetext{
"Plotly Technologies Inc. Collaborative data science. Montréal, QC, 2015. https://plot.ly.

** http://www.gemini.edu/sciops/telescopes-and-sites/observing-condition-constraints/ir-transmission-spectra

${ }^{\dagger \dagger}$ http://www.gemini.edu/sciops/instruments/nearir-resources/wavelength-calibration

${ }^{\ddagger \ddagger}$ https://www.eso.org/observing/dfo/quality/UVES/
} 


\section{REFERENCES}

[1] Woosley, S. E. and Bloom, J. S., "The Supernova Gamma-Ray Burst Connection," Annual Review of Astronomy and Astrophysics 44, 507-556 (Sep 2006).

[2] Hjorth, J., Malesani, D., Jakobsson, P., Jaunsen, A. O., Fynbo, J. P. U., Gorosabel, J., Krühler, T., Levan, A. J., Michałowski, M. J., Milvang-Jensen, B., Møller, P., Schulze, S., Tanvir, N. R., and Watson, D., "The Optically Unbiased Gamma-Ray Burst Host (TOUGH) Survey. I. Survey Design and Catalogs," The Astrophysical Journal 756, 187 (Sep 2012).

[3] Berger, E., "Short-Duration Gamma-Ray Bursts," Annual Review of Astronomy and Astrophysics 52, 43105 (Aug 2014).

[4] Fynbo, J. P. U., Watson, D., Thöne, C. C., Sollerman, J., Bloom, J. S., Davis, T. M., Hjorth, J., Jakobsson, P., Jørgensen, U. G., Graham, J. F., Fruchter, A. S., Bersier, D., Kewley, L., Cassan, A., Castro Cerón, J. M., Foley, S., Gorosabel, J., Hinse, T. C., Horne, K. D., Jensen, B. L., Klose, S., Kocevski, D., Marquette, J.-B., Perley, D., Ramirez-Ruiz, E., Stritzinger, M. D., Vreeswijk, P. M., Wijers, R. A. M., Woller, K. G., Xu, D., and Zub, M., "No supernovae associated with two long-duration $\gamma$-ray bursts," Nature 444, 1047-1049 (Dec 2006).

[5] Kann, D. A., Schady, P., Olivares E., F., Klose, S., Rossi, A., Perley, D. A., Krühler, T., Greiner, J., Nicuesa Guelbenzu, A., Elliott, J., Knust, F., Filgas, R., Pian, E., Mazzali, P., Fynbo, J. P. U., Leloudas, G., Afonso, P. M. J., Delvaux, C., Graham, J. F., Rau, A., Schmidl, S., Schulze, S., Tanga, M., Updike, A. C., and Varela, K., "Highly luminous supernovae associated with gamma-ray bursts. I. GRB 111209A/SN 2011kl in the context of stripped-envelope and superluminous supernovae," Astronomy \& Astrophysics 624, A143 (Apr 2019).

[6] Thöne, C. C., de Ugarte Postigo, A., Fryer, C. L., Page, K. L., Gorosabel, J., Aloy, M. A., Perley, D. A., Kouveliotou, C., Janka, H. T., Mimica, P., Racusin, J. L., Krimm, H., Cummings, J., Oates, S. R., Holland, S. T., Siegel, M. H., de Pasquale, M., Sonbas, E., Im, M., Park, W. K., Kann, D. A., Guziy, S., García, L. H., Llorente, A., Bundy, K., Choi, C., Jeong, H., Korhonen, H., Kubànek, P., Lim, J., Moskvitin, A., MuñozDarias, T., Pak, S., and Parrish, I., "The unusual $\gamma$-ray burst GRB 101225A from a helium star/neutron star merger at redshift 0.33," Nature 480, 72-74 (Dec 2011).

[7] de Ugarte Postigo, A., Blazek, M., Janout, P., Sprimont, P., Thöne, C. C., Gorosabel, J., and SánchezRamírez, R., "GRBSpec: a multi-observatory database for gamma-ray burst spectroscopy," in [Society of Photo-Optical Instrumentation Engineers (SPIE) Conference Series], Society of Photo-Optical Instrumentation Engineers (SPIE) Conference Series 9152 (July 2014).

[8] Zeh, A., Klose, S., and Kann, D. A., "Gamma-Ray Burst Afterglow Light Curves in the Pre-Swift Era: A Statistical Study," The Astrophysical Journal 637, 889-900 (Feb. 2006).

[9] Kann, D. A., Klose, S., and Zeh, A., "Signatures of Extragalactic Dust in Pre-Swift GRB Afterglows," The Astrophysical Journal 641, 993-1009 (Apr. 2006).

[10] Kann, D. A., Klose, S., Zhang, B., Malesani, D., Nakar, E., Pozanenko, A., Wilson, A. C., Butler, N. R., Jakobsson, P., Schulze, S., Andreev, M., Antonelli, L. A., Bikmaev, I. F., Biryukov, V., Böttcher, M., Burenin, R. A., Castro Cerón, J. M., Castro-Tirado, A. J., Chincarini, G., Cobb, B. E., Covino, S., D'Avanzo, P., D'Elia, V., Della Valle, M., de Ugarte Postigo, A., Efimov, Y., Ferrero, P., Fugazza, D., Fynbo, J. P. U., Gålfalk, M., Grundahl, F., Gorosabel, J., Gupta, S., Guziy, S., Hafizov, B., Hjorth, J., Holhjem, K., Ibrahimov, M., Im, M., Israel, G. L., Jeĺinek, M., Jensen, B. L., Karimov, R., Khamitov, I. M., Kiziloğlu, Ü., Klunko, E., Kubánek, P., Kutyrev, A. S., Laursen, P., Levan, A. J., Mannucci, F., Martin, C. M., Mescheryakov, A., Mirabal, N., Norris, J. P., Ovaldsen, J. E., Paraficz, D., Pavlenko, E., Piranomonte, S., Rossi, A., Rumyantsev, V., Salinas, R., Sergeev, A., Sharapov, D., Sollerman, J., Stecklum, B., Stella, L., Tagliaferri, G., Tanvir, N. R., Telting, J., Testa, V., Updike, A. C., Volnova, A., Watson, D., Wiersema, K., and $\mathrm{Xu}, \mathrm{D}$., "The Afterglows of Swift-era Gamma-ray Bursts. I. Comparing pre-Swift and Swift-era Long/Soft (Type II) GRB Optical Afterglows," The Astrophysical Journal 720, 1513-1558 (Sept. 2010).

[11] Kann, D. A., Klose, S., Zhang, B., Covino, S., Butler, N. R., Malesani, D., Nakar, E., Wilson, A. C., Antonelli, L. A., Chincarini, G., Cobb, B. E., D'Avanzo, P., D’Elia, V., Della Valle, M., Ferrero, P., Fugazza, D., Gorosabel, J., Israel, G. L., Mannucci, F., Piranomonte, S., Schulze, S., Stella, L., Tagliaferri, G., and Wiersema, K., "The Afterglows of Swift-era Gamma-Ray Bursts. II. Type I GRB versus Type II GRB Optical Afterglows," The Astrophysical Journal 734, 96 (June 2011). 
[12] Wells, D. C., Greisen, E. W., and Harten, R. H., "FITS - a Flexible Image Transport System," Astronomy E Astrophysics Supp. Ser. 44, 363W (1981).

[13] Vernet, J., Dekker, H., D'Odorico, S., Kaper, L., Kjaergaard, P., Hammer, F., Randich, S., Zerbi, F., Groot, P. J., Hjorth, J., Guinouard, I., Navarro, R., Adolfse, T., Albers, P. W., Amans, J. P., Andersen, J. J., Andersen, M. I., Binetruy, P., Bristow, P., Castillo, R., Chemla, F., Christensen, L., Conconi, P., Conzelmann, R., Dam, J., de Caprio, V., de Ugarte Postigo, A., Delabre, B., di Marcantonio, P., Downing, M., Elswijk, E., Finger, G., Fischer, G., Flores, H., François, P., Goldoni, P., Guglielmi, L., Haigron, R., Hanenburg, H., Hendriks, I., Horrobin, M., Horville, D., Jessen, N. C., Kerber, F., Kern, L., Kiekebusch, M., Kleszcz, P., Klougart, J., Kragt, J., Larsen, H. H., Lizon, J. L., Lucuix, C., Mainieri, V., Manuputy, R., Martayan, C., Mason, E., Mazzoleni, R., Michaelsen, N., Modigliani, A., Moehler, S., Møller, P., Norup Sørensen, A., Nørregaard, P., Péroux, C., Patat, F., Pena, E., Pragt, J., Reinero, C., Rigal, F., Riva, M., Roelfsema, R., Royer, F., Sacco, G., Santin, P., Schoenmaker, T., Spano, P., Sweers, E., Ter Horst, R., Tintori, M., Tromp, N., van Dael, P., van der Vliet, H., Venema, L., Vidali, M., Vinther, J., Vola, P., Winters, R., Wistisen, D., Wulterkens, G., and Zacchei, A., "X-shooter, the new wide band intermediate resolution spectrograph at the ESO Very Large Telescope," Astronomy \& Astrophysics 536, A105 (Dec 2011).

[14] Christensen, L., Fynbo, J. P. U., Prochaska, J. X., Thöne, C. C., de Ugarte Postigo, A., and Jakobsson, P., "A High Signal-to-noise Ratio Composite Spectrum of Gamma-ray Burst Afterglows," The Astrophysical Journal 727, 73 (Feb 2011). 


\section{morre}

Catalogue of GRB spectra Information about a specific spectrum.

Spectrum of GRB 170202A

\begin{tabular}{|l|l|}
\hline Mean time since burst (t-t0) : & 5.293 hours \\
\hline Exposure : & $3 \times 900$ seconds \\
\hline Spectral Range : & $3700-7800$ Á \\
\hline Slit width : & 1 \\
\hline Position Angle : & Paralactic \\
\hline Dispersing element : & R1000B \\
\hline Vacuum wavelengths : & No \\
\hline Heliocentric correction: & No \\
\hline Telluric correction: & No \\
\hline Redshift : & 3.645 \\
\hline Redshift : & 3.077 \\
\hline InstrumentTelescope : & OSIRIS / 10.4m Gran Telescopio Canarias (Spain / La Palma) \\
\hline Uploader : & Antonio de Ugarte Postigo, IAA-CSIC \\
\hline Modily Spectia & \\
\hline
\end{tabular}

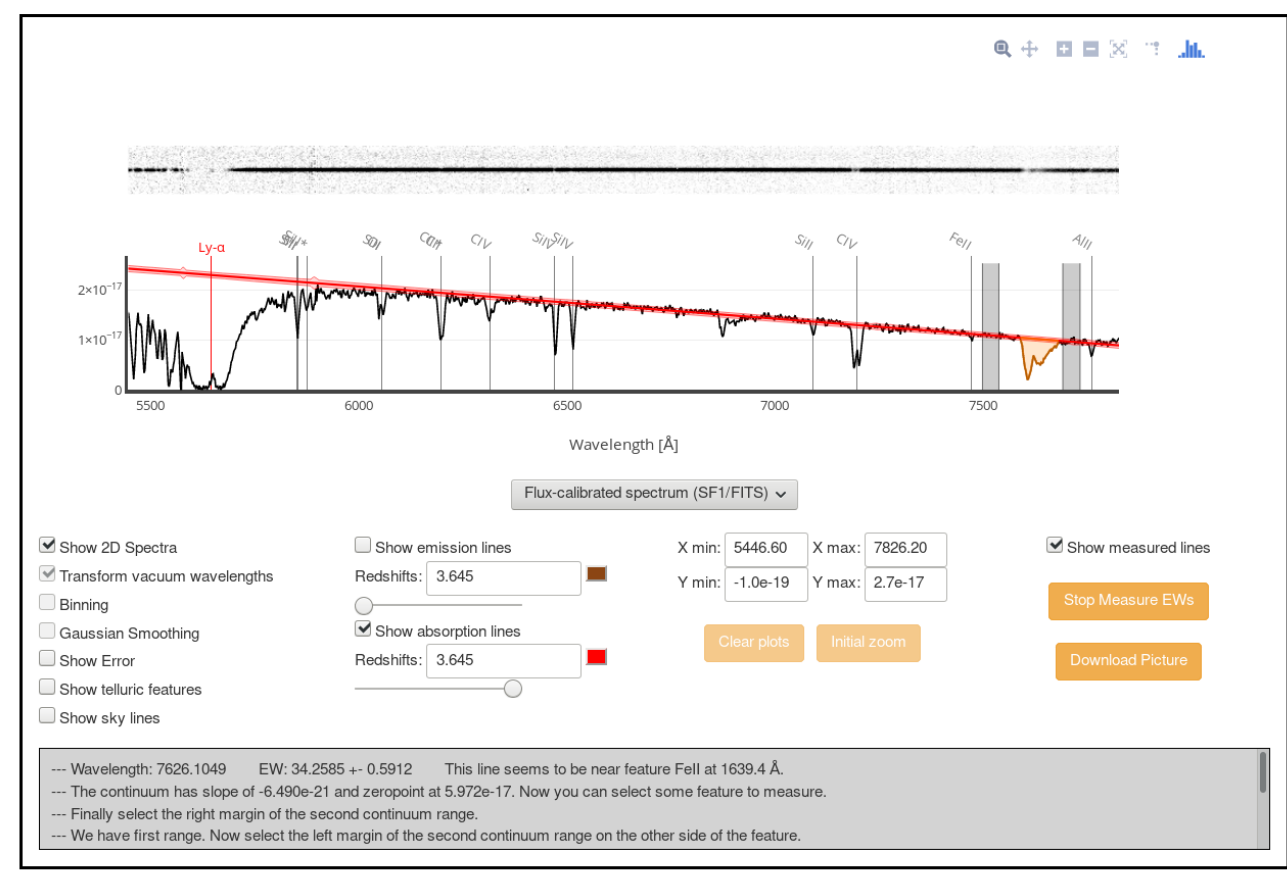

Equivalent widths:

\begin{tabular}{|c|c|c|c|c|c|c|}
\hline \multirow{2}{*}{$\begin{array}{l}\text { Observed } \lambda(\dot{A}) \text { : } \\
7626.10\end{array}$} & \multicolumn{3}{|l|}{ EW: } & \multicolumn{2}{|l|}{ Due to: } & $\mathrm{z}:$ \\
\hline & 34.26 & \pm & 0.59 & Fell 1639.4 & - & 3.645 \\
\hline
\end{tabular}

Figure 2. Example of a spectrum of GRB 170202A in the GRBSpec database (Part A). 


\begin{tabular}{|c|c|c|c|c|}
\hline Observed $\lambda(\dot{A})$ & Feature & $z$ & $\mathrm{EW}(\dot{\mathbf{A}})$ & Optlons \\
\hline 4266.64 & HI 1215.67 & 3.645 & $0 \pm 0$ & $+x$ \\
\hline \multirow[t]{2}{*}{5853.17} & SII 1259.52 & 3.645 & $1.649 \pm 0.127$ & $+x$ \\
\hline & Sill 1260.42 & 3.645 & & $\Delta x$ \\
\hline 5877.04 & Sill* 1264.74 & 3.645 & $0.94 \pm 0.197$ & +8 \\
\hline \multirow[t]{2}{*}{6054.85} & OI 1302.17 & 3.645 & $2.946 \pm 0.159$ & $+\infty x$ \\
\hline & Sill 1304.37 & 3.645 & & $x$ \\
\hline \multirow[t]{2}{*}{6198.74} & CII 1334.53 & 3.645 & $6.646 \pm 0.164$ & $+\infty x$ \\
\hline & CII* 1335.71 & 3.645 & & $x$ \\
\hline 6316.41 & CIV 1550.77 & 3.07308 & $3.472 \pm 0.201$ & $+\infty x$ \\
\hline 6470.07 & SilV 1393.76 & 3.64217 & $5.856 \pm 0.213$ & $+x$ \\
\hline 6514.73 & SilV 1402.77 & 3.64419 & $4.38 \pm 0.131$ & $+\infty$ \\
\hline 7091.69 & Sill 1526.71 & 3.64508 & $1.674 \pm 0.13$ & $+\infty x$ \\
\hline \multirow[t]{2}{*}{7195.44} & CIV 1548.2 & 3.645 & $13.634 \pm 0.228$ & +8 \\
\hline & CIV 1550.77 & 3.645 & & 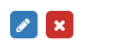 \\
\hline 7471.56 & Fell 1608.45 & 3.645 & $0.757 \pm 0.184$ & $+\infty x$ \\
\hline 7761.86 & Alll 1670.79 & 3.645 & $2.187 \pm 0.395$ & +6 \\
\hline
\end{tabular}

Import JSON formatted lines

Browse Upload

Measured fluxes :

No flux measurements are available

\begin{tabular}{|c|c|c|c|c|c|c|}
\hline Observed $\lambda(\dot{A})$ : & FLUX: & & & Due to: & & Z: \\
\hline 5364.35 & 6.37 & \pm & 0.53 & unknown 0 & $\cdot$ & 2.21 \\
\hline
\end{tabular}

Measured column densities :

No column density measurements are available.

\begin{tabular}{|c|c|c|c|c|c|c|c|}
\hline Element: & z: & column & g N: & & B: & & \\
\hline AllI & 2.21 & 6.37 & \pm & 0.53 & 6.37 & \pm & 0.53 \\
\hline
\end{tabular}

\section{Add $\log N$}

Spectrum files :

\begin{tabular}{|l|l|l|l|}
\hline Code & Flle & Unlts & Optlons \\
\hline SF1 & 1D flux-calibrated spectrum (fits) & $\dot{A}$ & 国 \\
\hline EF1 & 1D flux-calibrated error spectrum (fits) & $\dot{A}$ & 圆 \\
\hline SF2 & 2D flux-calibrated spectrum (fits) & $\dot{A}$ & 固 $\times$ \\
\hline
\end{tabular}

1D normalised spectrum (fits) -

Upload

Other spectra in same dataset :

\begin{tabular}{|l|l|l|l|l|l|}
\hline Optlons & t-to & Exposure & Range & Res. & Instrument/Telescope \\
\hline S Spectrum & 5.968 hours & $2 \times 900 \mathrm{~s}$ & $5100-9300 \mathrm{~A}$ & & OSIRIS / GTC \\
\hline
\end{tabular}

Figure 3. Example of a spectrum of GRB 170202A in the GRBSpec database (Part B). 